\title{
Primario desconocido de cabeza y cuello, una visión del oncólogo radioterapeuta
}

\begin{abstract}
*Correspondencia:
milerivero_4@hotmail.com

Departamento de Radioterapia Hospital de clínicas Dr. Manuel Quintela. Av. Italia, 11600. Montevideo - Uruguay
\end{abstract}

\section{Conflicto de intereses: Los} autores declaran no tener conflictos de intereses.

Recibido: 8 de Agosto 2021

Aceptado: 23 de Noviembre, 2021

Publicado: 20 de Diciembre, 2021

Editor: Dr. Felipe Campoverde

Membrete bibliográfico:

Rivero M, Lorenzo F, Torres $M$, Quarneti A. C. Primario desconocido de cabeza y cuello, una visión del oncólogo radioterapeuta. Rev. Oncol. Ecu 2021;31(3):234-242.

ISSN: 2661-6653

DOI: https://doi.org/10.33821/593

Copyright Rivero $\mathrm{M}$, et al. Este artículo es distribuido bajo los términos de Creative Commons Attribution License BY-NCSA 4.0, el cual permite el uso y redistribución citando la fuente y al autor original.

\section{Unknown primary of the head and neck, a vision of the radiation oncologist}

\author{
Milexys Rivero*1(i), Federico Lorenzo1, Marcelo Torres1, Aldo Quar- \\ neti1
}

1. Cátedra de Oncología Radioterápica, Universidad de la República, MontevideoUruguay.

\section{Resumen}

Se define carcinoma de cabeza y cuello (CCC) de primario desconocido al cuadro de adenopatía cervical en que, luego de examen físico, estudios de imágenes y panendoscopia con biopsias, no se encuentra el tumor primario pero sí la confirmación de malignidad de la adenomegalia. Son infrecuentes, por lo que estudios prospectivos que arrojen resultados estadísticamente significativos no están disponibles actualmente, y el tratamiento definitivo es aún motivo de controversia. Al ser la radioterapia un tratamiento dirigido es imprescindible definir adecuadamente los volúmenes blanco de tratamiento; es ideal el hallazgo del tumor primario, pero en muchos casos a pesar de un estudio escalonado, exhaustivo y multidisciplinar esto no se logra. Esto motiva el debate de qué regiones tratar, dosis, fraccionamiento y modalidad (exclusiva, adyuvante, en concurrencia). Hasta el momento el tratamiento de ganglios cervicales y mucosa de alto riesgo parece ser la estrategia con mejor control locorregional.

\section{Palabras claves:}

DeCS: Adenopatía cervical, cáncer de cabeza y cuello, carcinoma de células escamosas, radioterapia, quimioterapia.

DOI: $10.33821 / 593$ 


\begin{abstract}
Head and neck carcinoma (HNC) of unknown primary is a clinical condition defined as a cervical adenopathy for which, after physical examination, imaging studies and panendoscopy with biopsies, the primary tumor is not found, but there is confirmed malignancy of the adenomegaly. It is infrequent, so prospective studies that yield statistically significant results are not currently available, and definitive treatment is still controversial. Since radiation therapy is a targeted treatment, it is essential to adequately define treatment target volumes; the discovery of the primary tumor is ideal, but in many cases, despite a phased, exhaustive and multidisciplinary study, this is not achieved. This motivates the debate on which regions to treat, dose, fractionation and modality (exclusive, adjuvant, concurrent). Until now, the treatment of high-risk cervical nodes and mucosa seems to be the strategy with the best locoregional control.
\end{abstract}

\title{
Keywords:
}

MESH: Cervical adenopathy, head and neck cancer, squamous cell carcinoma, radiotherapy, chemotherapy.

DOI: $10.33821 / 593$

\section{Introducción}

El cáncer de cabeza y cuello de primario desconocido comprende la adenopatía cervical en que luego de un estudio exhaustivo no es posible esclarecer el tumor primario. Tiene baja incidencia entre los carcinomas de cabeza y cuello, aproximadamente un 3\% [1].

Los ganglios del nivel II son los más comúnmente afectados, asociados principalmente a primarios en la orofaringe, hipofaringe o laringe. Los ganglios linfáticos retrofaríngeos generalmente se consideran el primer escalón de la nasofaringe. No obstante, una adenopatía a cualquier nivel no encierra un diagnóstico [2].

Para confirmar malignidad se requiere una muestra del ganglio para estudio citológico o histológico, generalmente mediante punción y aspiración con aguja fina (PAAF) guiada por ecografía, la cual tiene alta sensibilidad, positiva en un $80 \%$ o más de los pacientes estudiados. Los casos de falsos negativos pueden deberse a la zona en que fue tomada la muestra, que pueden tener baja celularidad del líquido si fue dentro de una metástasis quística, en un área de necrosis, en una zona de inflamación peritumoral o incluso en zona de alta vascularización que da como resultado una muestra con sangre, situaciones ante las que se sugiere repetir la PAAF [3]. El carcinoma de células escamosas es la histología más común, seguido del adenocarcinoma [4].

Actualmente se recomienda estudios para el virus del papiloma humano (VPH) y el virus de Epstein-Barr (VEB), que sugieren primarios en orofaringe o nasofaringe, respectivamente. Incluso los marcadores para tiroides y pulmón son sugeridos, estos últimos principalmente en adenomegalias supraclaviculares e histología adenocarcinoma [5]. 


\section{Búsqueda de neoplasia primaria}

Se inicia la búsqueda con laringoscopia flexible y tomografía computada (TC) o resonancia magnética (RM) de cabeza y cuello. Sitios como base de lengua y amígdalas son los más difíciles de valorar por su tejido linfoide, con posibles falsos negativos.

Se complementará con estudios de extensión, el más utilizado la TC de tórax, abdomen y pelvis con contraste. Ante imágenes convencionales negativas la tomografía por emisión de positrones (PET, por sus siglas en inglés), ha sido útil en la ubicación del primario. La fusión del PET con la TC aumenta la sensibilidad con mayor definición anatómica [ 6 ], lo que ha reportado el primario en un $29 \%$ de los pacientes con imágenes convencionales previas negativas, alcanzando una sensibilidad y especificidad del $64.3 \%$ y $94.1 \%$, respectivamente. Miller et al. analizaron pacientes con adenopatías cervicales y encontraron que un $24 \%$ tenían tumores primarios por debajo de la clavícula en el PET-CT, siendo el sitio más común el pulmón, algo que cambia completamente el plan de tratamiento [7].

La panendoscopia está indicada posterior a la imágenes diagnósticas con la intención de biopsiar zonas sospechosas y confirmar el diagnóstico. Las biopsias aleatorias son controversiales; algunas guías, como la NCCN (National Comprehensive Cancer Network) y ASCO (American Society of Clinical Oncology) no la recomiendan por su bajo rendimiento. Un estudio de PET negativo en pacientes con un carcinoma primario oculto de cabeza y cuello no excluye la necesidad de una panendoscopia con biopsia para detectar el tumor primario oculto [6]. Sokoya et al. analizaron 103 pacientes cuyas exploraciones PET-CT fueron negativas para un tumor primario, y gracias a la panendoscopia en 32 pacientes estos primarios fueron identificados. El cáncer de amígdala representó el 56\%, el 25\% se ubicaron en base de la lengua, el 3\% en nasofaringe y el 3\% en hipofaringe [8].

Otra conducta adoptada es la amigdalectomía palatina, que puede ser ipsilateral o bilateral, ya que hay reportes de hasta un 10\% de primarios en la amígdala contralateral [9]. No se recomienda la biopsia de la amígdala como una opción a la amigdalectomía, pues algunos tumores son pequeños y están ubicados dentro de las criptas amigdalares por lo que no pueden identificarse mediante biopsia [10]. Si en este punto aún no se ha encontrado el sitio primario hemos llegado al diagnóstico de un primario desconocido de cabeza y cuello.

\section{Manejo multidisciplinario y tratamiento radiante}

La mejor decisión terapéutica se toma en un equipo multidisciplinario. Entre las opciones actualmente están la disección ganglionar del cuello, radioterapia y/o quimioterapia. La controversia aún es: ¿qué opción es mejor?, ¿el tratamiento debe ser único o combinado?

La cirugía del cuello además de ser una opción terapéutica aporta datos que complementan la estadificación ganglionar, pues se ha informado hasta un 30\% de infiltración ganglionar microscópica [11], por lo cual es ampliamente recomendada por la guía de NCCN, pero tiene limitantes como modalidad única.

Un estudio retrospectivo evaluó los resultados en pacientes con cáncer de cabeza y cuello de primario desconocido tratados en 5 centros de Dinamarca. Las opciones de tratamiento fueron cirugía de cuello sin adyuvancia, radioterapia ipsilateral en cuello o radioterapia bilateral en cuello y mucosas de alto riesgo. La cirugía como tratamiento único se asoció 
a un peor resultado [12]. Aunque este y muchos estudios tienen sesgos y grupos heterogéneos de pacientes, los resultados coinciden en que el factor pronóstico más importante es el compromiso ganglionar avanzado (N2-N3 de AJCC) y la extensión extracapsular (EEC). Otra serie encontró que la disección ganglionar del cuello más terapia adyuvante y la quimioradioterapia concurrente presentan tasas de control local y sobrevida libre de enfermedad mejores que la modalidad de tratamiento único, sea cirugía o radioterapia sola, principalmente en pacientes N3 o con extensión extracapsular [13].

Algunos estudios sugieren en pacientes N1 disección con observación, pero la evidencia no es sólida al respecto, por lo que pacientes N1-N2 deberían ser tratados con cirugía y radioterapia adyuvante, o radioquimioterapia concurrente [14]. Se ha reportado persistencia de la enfermedad ganglionar hasta en un $44 \%$ de los pacientes que se han sometido a disección ganglionar cervical luego de radioterapia, con una peor sobrevida y con mayor morbilidad postoperatoria en comparación con la cirugía seguida de radioterapia, por lo que se prefiere esta última secuencia [15].

Hasta el momento no está claro el beneficio de la quimioterapia en estos pacientes pero en el caso de enfermedad avanzada N3 se prefiere la radioquimioterapia [16]. La extensión extracapsular es una indicación para agregar quimioterapia a la radioterapia adyuvante, por su mayor riesgo de recurrencia, independiente del número y tamaño de los ganglios.

Siguiendo el patrón de drenaje linfático los niveles cervicales II, III y IV son seccionados, además de aquellos con enfermedad macroscópica.

En pacientes tratados con radioterapia, el debate es el campo de tratamiento: ¿se debe tratar cuello bilateral?, ¿se debe incluir la mucosa?

El drenaje linfático es principalmente ipsilateral, con baja incidencia contralateral, pero estructuras como paladar blando, base de la lengua, pared faríngea posterior, laringe y nasofaringe tienen drenaje bilateral [11]. No está demostrado si la radioterapia en toda la mucosa faríngea y en ambos lados del cuello mejora la sobrevida versus un campo de tratamiento reducido. Los estudios que comparan la irradiación del cuello unilateral con la bilateral han mostrado resultados contradictorios. Series retrospectivas que informaron sobre pacientes tratados con radioterapia cervical unilateral, encontraron que la recurrencia ganglionar cervical contralateral era baja, tasas entre el 2 y el 10\% [17].

Con esta baja evidencia es difícil favorecer una estrategia sobre otra, pero parece lógico extrapolar el tratamiento del cuello de un primario conocido, en los que usualmente se trata el cuello bilateral. Es conveniente y poco discutida la necesidad de irradiación bilateral ante N3-N2, pero en el caso de pacientes N1, este aspecto es menos claro, excepto ante sospecha de primario en nasofaringe, donde típicamente se trata el cuello bilateral. Una opción válida sería irradiar el cuello en base a los resultados del PET-CT. Ante la no disponibilidad de este método, pacientes N1 con compromiso de un ganglio del nivel II, luego de disección, vaciamiento mínimo de 10 ganglios, sin EEC, ni invasión linfovascular pueden ser tratados con radioterapia adyuvante unilateral, incluyendo los niveles II a IV, [18] tratando de disminuir las dosis en tejido sano.

Un metaanálisis que incluyó 33 estudios con cáncer de cabeza y cuello de primario desconocido encontró una menor tasa de recurrencia al irradiar cuello y mucosa de alto riesgo versus solo cuello. Los resultados fueron $12 \%$ versus $16 \%$ a favor del tratamiento de las mucosas [19].

Un estudio retrospectivo realizado en China incluyó 154 pacientes tratados con radioterapia. Las tasas de supervivencia global (SG) y supervivencia libre de progresión (SLP) a 5 
años fueron $64.2 \%$ y $87.3 \%$, respectivamente. La radioterapia de la mucosa faríngea se asociaba con una mejor SG, pero no con mejor SLP. Al comparar radioterapia 3D vs IMRT la diferencia radicó en menor toxicidades (disfagia, xerostomía, disfagia o fibrosis cutánea) con IMRT [20]. Resultados concordantes con otras series [21].

Estos datos sugieren que la irradiación de la mucosa reduce significativamente el riesgo de falla del sitio primario o más específicamente, que surja una lesión primaria luego del tratamiento [17].

Bhide et al demostraron el beneficio del uso de IMRT en pacientes con carcinoma de células escamosas de cabeza y cuello con sitio primario oculto, con mayor homogeneidad de la dosis y menor dosis en parótidas [22]. Pacientes tratados con IMRT, con dosis en mucosa de alto riesgo entre 56-66 Gy, enfermedad ganglionar macroscópica 70 Gy o adyuvancia en cuello 66 Gy, fueron seguidos por 2 años y reportaron tasas de sobrevida libre de progresión locorregional mayores al $90 \%$, un $25 \%$ de xerostomía grado 2, pero alta incidencia de estenosis esofágica, alrededor del 40\% [23]. Otros estudios que utilizaron VMAT e IMRT concordaron en varios puntos, pero menor tasa de estenosis esofágica (5.9\%) [16].

Se asume entonces que el tratamiento de la mucosa es necesario y que debería ser con técnicas avanzadas de radioterapia. Pero ¿debería ser irradiada toda la mucosa faríngea o la considerada de alto riesgo paciente específico?

Datos actuales sugieren que la mayoría de los tumores de cabeza y cuello de primario desconocido probablemente se localizan en orofaringe, subsitio amígdala o base de lengua [16]. Un enfoque especial es la nasofaringe en zonas endémicas, fuertemente asociado a la presencia de VEB, la cual sería otra mucosa de alto riesgo. Series retrospectivas en pacientes no asiáticos, han reportado resultados satisfactorios en control local con la irradiación de la orofaringe como mucosa de alto riesgo, ganglios retrofaríngeos y cervicales bilaterales, sin tratar laringe, hipofaringe ni nasofaringe, algo que actualmente podría justificarse en pacientes $\mathrm{VPH} / \mathrm{p} 16$ positivos [24].

Un estudio prospectivo fase II realizado en china, seleccionó pacientes con CCC de primario oculto, comparando la irradiación completa de la mucosa faríngea o la irradiación electiva. En China se presenta una alta incidencia de carcinoma nasofaríngeo, por lo tanto, los pacientes se clasificaron como sospechoso de carcinoma nasofaríngeo si tenía compromiso de ganglios N II y retrofaríngeos o VEB positivo, de los contrario eran no sospechosos de carcinoma nasofaríngeo. El grupo sospechoso de se trató mediante irradiación electiva en la nasofaringe y el cuello bilateral; mientras el otro grupo se trató con disección del cuello seguida de irradiación unilateral del cuello para N1, N2a $<4 \mathrm{~cm} 0 \geq 4 \mathrm{~cm}$ sin EEC, o enfermedad N2b $<3$ ganglios positivos y dentro de un nivel ganglionar; para todos los demás casos se realizó irradiación bilateral del cuello, además la orofaringe unilateral, hipofaringe y estructuras supraglóticas; respetando la nasofaringe, la cavidad oral, las cuerdas vocales, la laringe subglótica y el esófago cervical. En una mediana de seguimiento de 76 meses ningún paciente experimentó un primario de CC. No se produjo toxicidad aguda o tardía de grado 4 . La toxicidad aguda de grado 3 más frecuente fue la mucositis oral (45.7\%), xerostomía y disfagia de grado 3 en $2(4.3 \%)$ y 4 pacientes (8.7\%), respectivamente [25].

Acerca de las dosis y fraccionamiento, las mismas se extrapolan de los tratamientos radiantes de un primario conocido. Partiendo de un fraccionamiento convencional (1.8 a 2 Gy por fracción) se prescribe a los sitios de la enfermedad macroscópica inicial 70 Gy, después de la resección en el lecho de los ganglios linfáticos 60 a 66 Gy (según hallazgos patológicos 
y la presencia de EEC, y en mucosa de alto riesgo 50 a 54 Gy. Dosis menores a 50 Gy se ha asociado a mayor falla local [16].

\section{Conclusiones}

Los CCC de primario desconocido presentan tasas de supervivencia global a 5 años similares a las de los CCC con un primario conocido. El tratamiento de modalidad combinada presenta buenas tasas de control locoregional. Por lo tanto, se debe también tener presente el riesgo de carcinomas radioinducidos cuando se tratan grandes volúmenes en pacientes con un buen pronóstico y larga expectativa de vida.

Por todo lo anterior, en pacientes que presentan adenopatía aislada de nivel II, particularmente con pruebas positivas para VEB, VPH o $\mathrm{p} 16$, se sugiere tratar orofaringe y nasofaringe, y a menudo se excluyen laringe e hipofaringe. Ante compromiso de los niveles ganglionares III y IV, especialmente en ausencia de adenopatía de nivel II, se considera que la laringe y la hipofaringe son sitios probablemente primarios y deben irradiarse.

Nota del Editor

La Revista Oncología Ecu permanece neutral con respecto a los reclamos jurisdiccionales en mapas publicados y afiliaciones institucionales.

\section{Abreviaturas}

VEB: virus de Epstein-Barr, VPH: virus del papiloma humano.

\section{Información administrativa}

Archivos Adicionales

Ninguno declarado por los autores.

Agradecimientos

No aplica.

Contribuciones de los autores

Milexys Rivero: conceptualización, validación, visualización, metodología, administración de proyecto, escritura: revisión y edición, curación de datos, análisis formal, adquisición de fondos, investigación, recursos, software, redacción -borrador original.

Federico Lorenzo: conceptualización, validación, visualización, metodología, administración de proyecto, escritura: revisión y edición, curación de datos, análisis formal.

Marcelo Torres: conceptualización, análisis formal, adquisición de fondos, investigación, recursos, software, redacción -borrador original.

Aldo Quarneti: conceptualización, validación, metodología, administración de proyecto, curación de datos, análisis formal, adquisición de fondos, investigación.

Todos los autores leyeron y aprobaron la versión final del manuscrito.

Financiamiento

Los autores realizaron el financiamiento de los gastos incurridos en la presente investigación. 


\section{Declaraciones}

Aprobación del comité de ética

No aplica.

Consentimiento para publicación

No aplica para estudios que no publican imágenes explícitas como tomografías, resonancias, imágenes de exámenes físicos.

Conflictos de interés

Los autores declaran que no tener ningún conflicto de competencia o interés.

Información de los autores

Milexys Rivero: Oncóloga Radioterápica Universidad de la República; Montevideo- Uruguay. https://orcid.org/00000002-1697-0904

Federico Lorenzo: Profesor adjunto cátedra Oncología Radioterápica Universidad de la República; Montevideo- Uruguay. https://orcid.org/0000-0002-8929-7685

Marcelo Torres: Asistente docente cátedra Oncología Radioterápica Universidad de la República; Montevideo- Uruguay. https://orcid.org/0000-0001-6262-9689

Aldo Quarneti: Profesor cátedra Oncología Radioterápica Universidad de la República; Montevideo- Uruguay. https://orcid.org/0000-0001-6273-4059.

\section{Referencias}

1. Cerezo L, Raboso E, Ballesteros Al. Unknown primary cancer of the head and neck: a multidisciplinary approach. Clin Trans/ Oncol. 2011; 13(2): 88-97. doi: 10.1007/s12094-011-0624-y

2. Grégoire V, Ang K, Budach W, Grau C, Hamoir M, Langendijk JA, et al. Delineation of the neck node levels for head and neck tumors: A 2013 update. DAHANCA, EORTC, HKNPCSG, NCIC CTG, NCRI, RTOG, TROG consensus guidelines. Radiother Oncol. 2014;110(1):172-81. doi: 10.1016/i.radonc. 2013.10.010

3. Tabet P, Saydy N, Letourneau-Guillon L, Gologan O, Bissada É, Ayad T, et al. Tabet JC, Guertin L, NguyenTan PF, Christopoulos A. Cystic masses of the lateral neck: Diagnostic value comparison between fineneedle aspiration, core-needle biopsy, and frozen section. Head \& neck. 2019;41(8):2696-2703. doi:: 10.1002/hed. 25755

4. Issing WJ, Taleban B, Tauber S. Diagnosis and management of carcinoma of unknown primary in the head and neck. Eur Arch Otorhinolaryngol. 2003;260(8):436-443. Disponible en: https://doi.org/10.1007/s00405-003-0585-z

5. Tandon S, Shahab R, Benton JI, Ghosh SK, Sheard J, Jones TM. Fine-needle aspiration cytology in a regional head and neck cancer center: comparison with a systematic review and meta-analysis. Head \& neck. 2008;30(9):1246-1252. doi: 10.1002/hed.20849 
6. Keller F, Psychogios G, Linke R, Lell M, Kuwert T, Iro H, Zenk J. Carcinoma of unknown primary in the head and neck: comparison between positron emission tomography (PET) and PET/CT. Head \& neck. 2011;33(11):1569-1575. doi: 10.1002/hed.21635

7. Miller FR, Hussey D, Beeram M, Eng T, McGuff HS, Otto RA. Positron emission tomography in the treatment of unknown primary carcinoma of the head and neck. Arch Otolaryngol. 2005;131:626-629. SU: jama

8. Sokoya M, Chowdhury F, Kadakia S, Ducic Y. Combination of panendoscopy and positron emission tomography/computed tomography increases detection of unknown primary head and neck carcinoma. The Laryngoscope. 2018;128(11):2573-2575. doi: $\underline{10.1002 / l a r y .27268}$

9. Koch WM, Bhatti N, Williams MF, Eisele DW. Oncologic rationale for bilateral tonsillectomy in head and neck squamous cell carcinoma of unknown primary source. Otolaryngology--head and neck surgery : official journal of American Academy of Otolaryngology-Head and Neck Surgery. 2001;124(3):331-333. doi: $\underline{10.1067 / \mathrm{mhn} .2001 .114309}$

10. Waltonen JD, Ozer E, Schuller DE, Agrawal A. Tonsillectomy vs. deep tonsil biopsies in detecting occult tonsil tumors. The Laryngoscope. 2009; 119(1):102-106. doi: 10.1002/lary.20017

11. Grégoire V, Coche E, Cosnard G, Hamoir M, Reychler H. Selection and delineation of lymph node target volumes in head and neck conformal radiotherapy. Proposal for standardizing terminology and procedure based on the surgical experience. Radiotherapy and Oncology. 2000; 56(2): 135-150. SU: thegreenjournal

12. Grau C, Johansen LV, Jakobsen J, Geertsen P, Andersen E, Jensen BB. Cervical lymph node metastases from unknown primary tumours. Results from a national survey by the Danish Society for Head and Neck Oncology. Radiotherapy and oncology : journal of the European Society for Therapeutic Radiology and Oncology. 2000; 55(2), 121-129. doi: 10.1016/s0167-8140(00)00172-9

13. Hung YH, Liu SA, Wang CC, Wang CP, Jiang RS, Wu SH. Treatment outcomes of unknown primary squamous cell carcinoma of the head and neck. 2018; 13(10): e0205365. doi: 10.1371/journal.pone.0205365

14. Radiotherapy dose fractionation, third edition. Royal College of Radiologists, 2019. BFCO(19)3.

15. Erkal HS, Mendenhall WM, Amdur RJ, Villaret DB, Stringer SP. Squamous cell carcinomas metastatic to cervical lymph nodes from an unknown mucosal site of the head and neck treated with radiation therapy alone or in combination with neck dissection. Int J Radiat Oncol Biol Phys. 2001;50(1):55-63. doi: 10.1016/s0360-3016(00)01554-6

16. Perkins SM, Spencer CR, Chernock RD, et al. Radiotherapeutic Management of Cervical Lymph Node Metastases From an Unknown Primary Site. Arch Otolaryngol Head Neck Surg. 2012;138(7):656-661. doi: 10.1001/archoto.2012.1110

17. Reddy SP, Marks JE. Metastatic carcinoma in the cervical lymph nodes from an unknown primary site: results of bilateral neck plus mucosal irradiation vs. ipsilateral neck irradiation. Int J Radiat Oncol Biol Phys. 1997; 37(4):797-802. doi: 10.1016/s0360-3016(97)00025-4

18. Biau J, Lapeyre M, Troussier I, Budach W, Giralt J, Grau C, Kazmierska J, Langendijk JA, Ozsahin M, O'Sullivan B, Bourhis J, Grégoire V. Selection of lymph node target volumes for definitive head and neck radiation therapy: a 2019 Update. Radiother Oncol. 2019;134:1-9. doi: 10.1016/j.radonc.2019.01.018

19. Liu X, Li D, Li N, Zhu X. Optimization of radiotherapy for neck carcinoma metastasis from unknown primary sites: a meta-analysis. Oncotarget. 2016;7(48):78736-78746. doi: 10.18632/oncotarget.12852.

20. Wang Y, He SS, Bao Y, Cai XY, Chen HY, Yang XL, Chen DM, Lu LX, Chen Y. Cervical lymph node carcinoma metastasis from unknown primary site: a retrospective analysis of 154 patients. Cancer Med. 2018; 7(5):1852-1859. doi: $10.1002 / \mathrm{cam} 4.1458$

21. Mendenhall WM, Mancuso AA, Amdur RJ, Stringer SP, Villaret DB, Cassisi NJ. Squamous cell carcinoma metastatic to the neck from an unknown head and neck primary site. American journal of otolaryngology. 2001; 22(4): 261-267. doi: 10.1053/ajot.2001.24820

22. Bhide S, Clark C, Harrington K, Nutting CM. Intensity modulated radiotherapy improves target coverage and parotid gland sparing when delivering total mucosal irradiation in patients with squamous cell carcinoma 
of head and neck of unknown primary site. Med Dosim. 2007;32(3):188-195. doi: 10.1016/j.meddos.2007.01.002

23. Sher DJ, Balboni TA, Haddad RI, Norris CM, Jr Posner MR, Wirth LJ, et al. Efficacy and toxicity of chemoradiotherapy using intensity-modulated radiotherapy for unknown primary of head and neck. International journal of radiation oncology, biology, physics. 2011;80(5):1405-1411. doi: 10.1016/j.jijobp. 2010.04.029

24. Mourad WF, Hu KS, Shasha D, Concert C, Ishihara D, Lin W, et al. Initial experience with oropharynx-targeted radiation therapy for metastatic squamous cell carcinoma of unknown primary of the head and neck. Anticancer Res. 2014; 34 (1): 243-248. SU: iiarjournals

25. Dou S, Li R, Zhang L, Wang Z, Xie L, Zhang C, Zhu G. Long-term results of elective mucosal irradiation for head and neck cancer of unknown primary in Chinese population: The EMICUP study. Cancer medicine. 2020; 9(5): 1712-1720. doi: $10.1002 /$ cam4.2856

DOI: Digital Object Identifier PMID: PubMeD Identifier SU: Short URL 